A-25-2020

\title{
EVALUACIÓN DEL USO DE RECURSOS HÍDRICOS NO CONVENCIONALES EN LA SALINIDAD DEL SUELO Y EL DESARROLLO DEL CULTIVO DE LECHUGA MEDIANTE EL USO DE DRONES EN EL CAMPO DE CARTAGENA
}

\section{Pedrero Salcedo, Francisco ${ }^{1}$, Llobet Escabias, Marina ${ }^{2}$, Boesveld, Harm ${ }^{2}$, Bartholomeus,} Harm $^{3}$, Alarcón Cabañero, Juan José ${ }^{1}$

${ }^{1}$ Departamento de Riego. Centro de Edafología y Biología Aplicada del Segura (CEBAS), Campus de Espinardo, N-25 Espinardo 30.100 (Murcia). fpedrero@cebas.csic.es

2 Water Resources Management Group. Wageningen university \& Research. Droevendaalsesteeg 4, 6708 PB Wageningen.

3 Geo-Information Science and Remote Sensing. Wageningen university \& Research. Droevendaalsesteeg 4, 6708 PB Wageningen.

\section{Introducción}

Una de las causas de la degradación del suelo es la salinidad, que afecta a mil millones de hectáreas en más de 100 países (Squires y Glenn, 2004), y se prevé que aumente 2 millones de hectáreas por año (Abbas et al., 2013). Por otro lado, el uso de fuentes de agua de riego no convencionales es necesario para reducir la presión sobre las fuentes de agua convencionales (ríos y acuíferos) para uso doméstico y satisfacer la futura demanda de alimentos, a la vez que es sostenible y económicamente viable. Por esta razón, es necesario establecer protocolos de buenas prácticas de gestión del riego a través de tecnologías agrícolas de precisión para evitar la salinización y utilizar correctamente los recursos hídricos no convencionales para el riego.

\section{Objetivo}

El objetivo de este trabajo es evaluar la salinidad del suelo y el desarrollo del cultivo de lechuga a través de imágenes térmicas y multiespectrales utilizando recursos hídricos no convencionales salinos en Murcia. La evaluación se realizó procesando imágenes térmicas y multiespectrales capturadas con dron bajo invernadero y en una parcela comercial al aire libre.

\section{Materiales y métodos}

Parcelas experimentales y diseño

El estudio se llevó a cabo en un invernadero del CEBAS-CSIC ubicado en el interior de la planta de tratamiento de aguas residuales de Roldán, Lo Ferro, Balsicas (Murcia) y en una parcela comercial al aire libre. El invernadero tiene una superficie de $680 \mathrm{~m}^{2}$, sistemas de calefacción, ventilación de pantalla, automatismos de control climático y sistemas de riego. Además, se estudió una parcela comercial al aire libre ubicada en La Aparecida (Murcia). En 
el ensayo bajo invernadero, la experiencia consistió en el cultivo de lechugas mini-romanas (Lactuca sativa var. Romana) en suelo. El trasplante se realizó el 24 de octubre de 2019 y la cosecha el 16 de diciembre de 2019. La distancia entre hileras fue de $15 \mathrm{~cm}$ y el marco de la plantación de $0,16 \times 1 \mathrm{~m}\left(6 \mathrm{p} / \mathrm{m}^{2}\right)$. Se utilizaron dos fuentes de agua distinta a partir del efluente producido en un prototipo de desalación de aguas residuales tratadas. Por una parte, se obtuvo un tratamiento salino (SP) mediante la mezcla del efluente y la salmuera producida en el prototipo $\left(C E=3-4 \mathrm{dS} \mathrm{m}^{-1}\right)$, y un segundo tratamiento con el efluente del prototipo $(\mathrm{CP}) \sin$ mezclar con salmuera $\left(\mathrm{CE}=0.7-0.8 \mathrm{dS} \mathrm{\textrm {m } ^ { - 1 }}\right.$ ) como tratamiento control. Para ambos tratamientos se realizaron 6 líneas con 60 plantas por línea.

La parcela comercial (IP) tenía un área de 1 ha de lechuga (variedad Mestiza). El trasplante tuvo lugar el 17 de octubre de 2019, con una densidad de plantación de 6,75/ $\mathrm{m}^{2}$, y la cosecha el 6 de enero de 2020. El agua de riego utilizada por el agricultor es el resultado de la combinación de diferentes fuentes de agua (lluvia, aguas regeneradas y aguas subterráneas) y se aplicó con un sistema de riego por goteo.

En ambas parcelas se realizaron análisis de agua, planta y suelo. Tres muestras de cada fuente de agua de riego fueron recolectada semanalmente durante los ciclos de cultivo para caracterizar la calidad del agua de riego. Las muestras se recogieron en botellas de vidrio y fueron transportadas en una hielera al laboratorio y almacenadas a $5{ }^{\circ} \mathrm{C}$ antes de ser procesadas para análisis químico. La concentración de $\mathrm{Na}, \mathrm{K}, \mathrm{Ca}, \mathrm{Mg}, \mathrm{Fe}, \mathrm{B}, \mathrm{Mn}, \mathrm{Ni}, \mathrm{Cu}, \mathrm{Zn}$ y los metales pesados $\mathrm{Cd}, \mathrm{Cr}$ y $\mathrm{Pb}$ fueron determinados por plasma acoplado inductivamente (ICP-ICAP 6500 DUO Thermo, Inglaterra). Aniones (cloruro, nitrato, fosfato y sulfato) fueron analizados por cromatografía iónica (Metrohm, Suiza) con un cromatógrafo de líquidos. Los mismos análisis fueron realizados para suelo y cultivo.

\section{Técnicas de teledetección y procesamiento de datos}

También se utilizaron técnicas de teledetección y procesamiento de datos para caracterizar ambas parcelas experimentales. Se utilizaron imágenes de dron para medir las respuestas espectrales y térmicas del suelo y el dosel a las condiciones salinas. Se realizaron un total de tres vuelos en cada parcela. El programa ArcGIS (versión 10.5) se utilizó para procesar las imágenes y calcular los diferentes índices. Para este estudio, se eligieron dos índices vegetativos (Índice de Vegetación de Diferencia Normalizada (NDVI) y la temperatura de la canopia) y dos índices de suelo (Índice de Salinidad del Suelo (SI) y el Índice de Salinidad de Diferencia Normalizada (NDSI)) para relacionarlos con la salinidad del suelo.

\section{Resultados y Discusión}

Las diferentes aguas de riego utilizadas en el ensayo tuvieron diferencias significativas en diferentes parámetros, especialmente los relacionados con la salinidad (Tabla 1). La Ta de la canopia mostró resultados prometedores, ya que obtuvo una buena correlación lineal de $\left(R^{2}\right.$ $=0.5$ ) y mostró el mismo patrón visual que la salinidad del suelo cuando la cámara térmica se utilizó en condiciones climáticas óptimas tanto en el ensayo bajo invernadero (Figura 1) como en el ensayo de la parcela comercial (Figura 2). Los resultados encontrados en esta tesis respaldan la teoría de que un cultivo menos tolerante mostrará diferencias de temperatura más altas y, por lo tanto, tendrá mejores correlaciones (Ivushkin et al., 2018; Tian et al., 2019). Además, el patrón de canopia $\mathrm{T}^{\mathrm{a}}$ y ECsat en T2 fue muy similar, lo que también se observó en un estudio anterior (Ivushkin et al., 2017). El otro índice vegetativo (NDVI) tenía correlaciones muy bajas y no hubo diferencias visuales aparte del tamaño más pequeño en las etapas iniciales de las lechugas cultivadas en la SP en comparación con las de la CP (datos no mostrados).

Tabla 1. Características químicas de las aguas de riego utilizadas en en ensayo bajo invernadero ( $\mathrm{CP}=$ efluente del prototipo, $\mathrm{SP}=$ tratamiento salino) y en la parcela comercial (IP) junto con la desviación estándar (DS). 


\begin{tabular}{|c|c|c|c|c|c|c|c|c|c|}
\hline \multirow{2}{*}{ parametros } & \multicolumn{3}{|c|}{$\mathrm{CP}$} & \multicolumn{3}{|c|}{ SP } & \multicolumn{3}{|c|}{ IP } \\
\hline & Media & DS & & Media & DS & & Media & DS & \\
\hline EC (dS/m) & 1 & 0,38 & $\mathrm{a}$ & 4,42 & 0,41 & $\mathrm{~b}$ & 1,01 & 0,11 & $\mathrm{a}$ \\
\hline pH & 8,02 & 0,13 & $\mathrm{a}$ & 7,77 & 0,15 & $a b$ & 8,50 & 0,43 & $\mathrm{a}$ \\
\hline $\mathrm{Cl}^{-}(\mathrm{mg} / \mathrm{l})$ & 322,11 & 368,19 & $\mathrm{a}$ & 861,1 & 361,6 & $\mathrm{~b}$ & 176,67 & 21,42 & $\mathrm{c}$ \\
\hline $\mathrm{NO}_{3}{ }^{-}(\mathrm{mg} / \mathrm{l})$ & 20,14 & 6,47 & $\mathrm{a}$ & 25,71 & 1,98 & $a b$ & 2,73 & 0,64 & $\mathrm{c}$ \\
\hline $\begin{array}{l}\mathrm{PO}_{4}{ }^{3-} \\
(\mathrm{mg} / \mathrm{l})\end{array}$ & 3,94 & 0,68 & $\mathrm{a}$ & 10,83 & 7,85 & $\mathrm{~b}$ & 3,18 & 0,90 & $\mathrm{a}$ \\
\hline $\begin{array}{l}\mathrm{SO}_{4}{ }^{2-} \\
(\mathrm{mg} / \mathrm{l}) \\
\end{array}$ & 92,14 & 63,37 & $\mathrm{a}$ & 319,1 & 185,27 & $\mathrm{~b}$ & 82,96 & 17,05 & $\mathrm{a}$ \\
\hline $\mathrm{Na}^{+}(\mathrm{mg} / \mathrm{l})$ & 152,36 & 43,5 & $\mathrm{a}$ & 762,36 & 47,41 & $\mathrm{~b}$ & 127,42 & 13,08 & $\mathrm{c}$ \\
\hline $\mathbf{P}(\mathrm{mg} / \mathrm{l})$ & 1,37 & 0,56 & $\mathrm{a}$ & 5,04 & 4,09 & $\mathrm{ab}$ & 1,00 & 0,48 & $\mathrm{a}$ \\
\hline $\mathrm{S}(\mathrm{mg} / \mathrm{l})$ & 43,22 & 19,76 & $\mathrm{a}$ & 149,86 & 96,94 & $\mathrm{~b}$ & 33,55 & 10,49 & $\mathrm{a}$ \\
\hline
\end{tabular}

Misma letra significa que no son significativamente diferentes de acuerdo con la prueba HSD de Tukey $(P<0.05)$

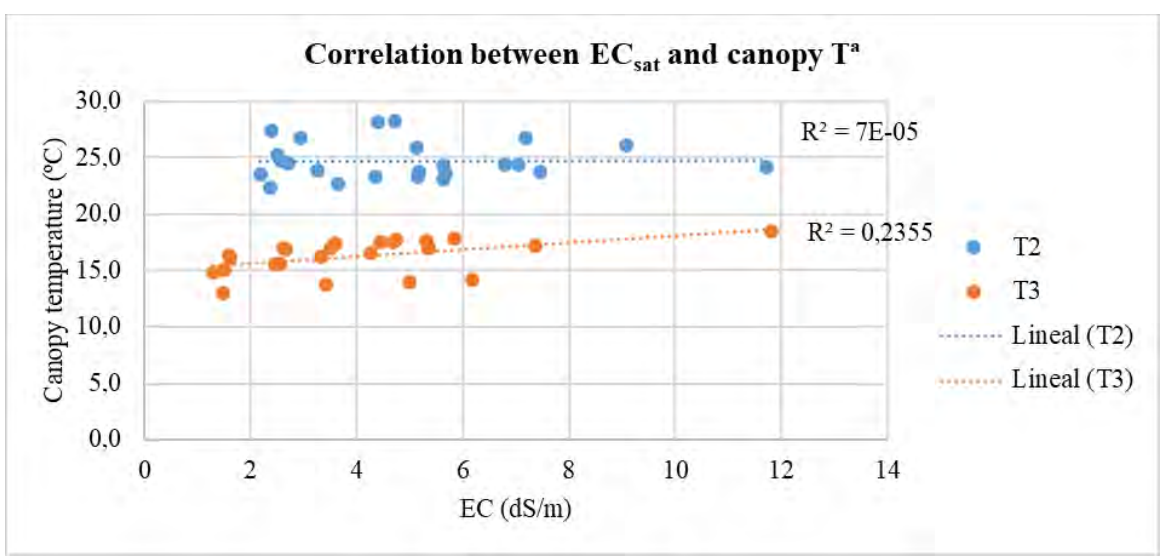

Figura 1. Correlación lineal entre el ECsat y la temperatura de la canopia para T2 $(12 / 02 / 2019)$ y T3 (16/12/2019)

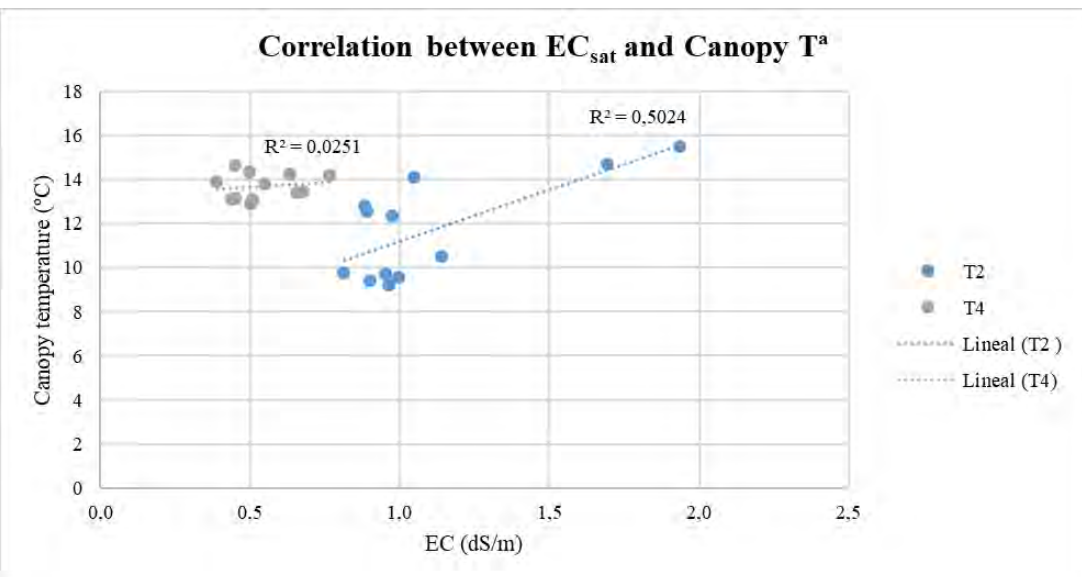

Figura 2. Correlación lineal entre la temperatura del dosel y la conductividad eléctrica del suelo para T2 (20/11/2019) y T4 (16/12/2019) 
Los índices de salinidad del suelo, SI y NDSI, obtuvieron buenas correlaciones, pero los patrones de salinidad del suelo no se distinguieron. De todos los índices, el que mejor se comportó fue el SI, con correlaciones lineales entre 0.63-0.83 tanto en el ensayo bajo invernadero (Figura 3,4) como en la parcela comercial (Figura 5,6). Se observaron resultados similares en estudios recientes como Gorji y col. (2017) donde monitorearon la salinidad del suelo en un lago salado y sus alrededores en Turquía, obteniendo una correlación de 0.77. Además, en otro estudio realizado en una zona agrícola en Marruecos (Lhissou et al., 2014), se encontró una correlación moderada $\left(R^{2}=0.55\right)$ entre SI y ECsat. Sin embargo, cuando se aplicó SI en suelos muy salinos, Allbed et al. (2014) obtuvieron correlaciones insignificantes y bajas en un oasis dominado con palmera datilera en Arabia Saudita.

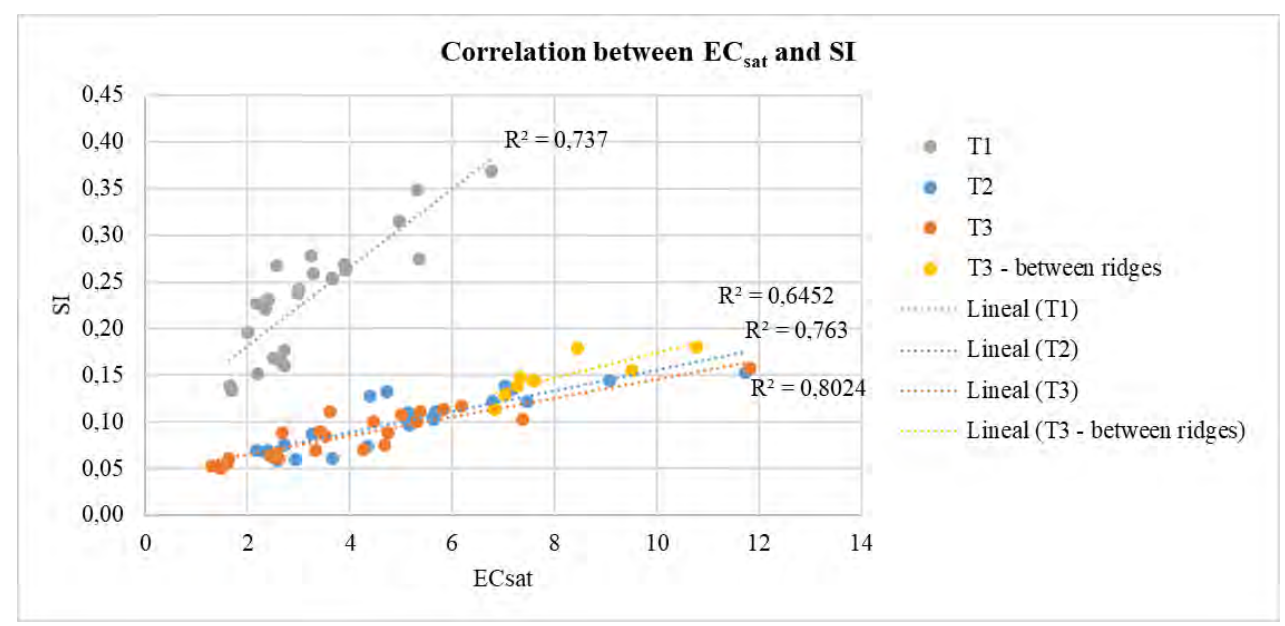

Figura 3. Correlación lineal entre el ECsat y el SI para T1 (12/12/2019), T2 (02/12/2019) y T3 $(16 / 12 / 2019)$
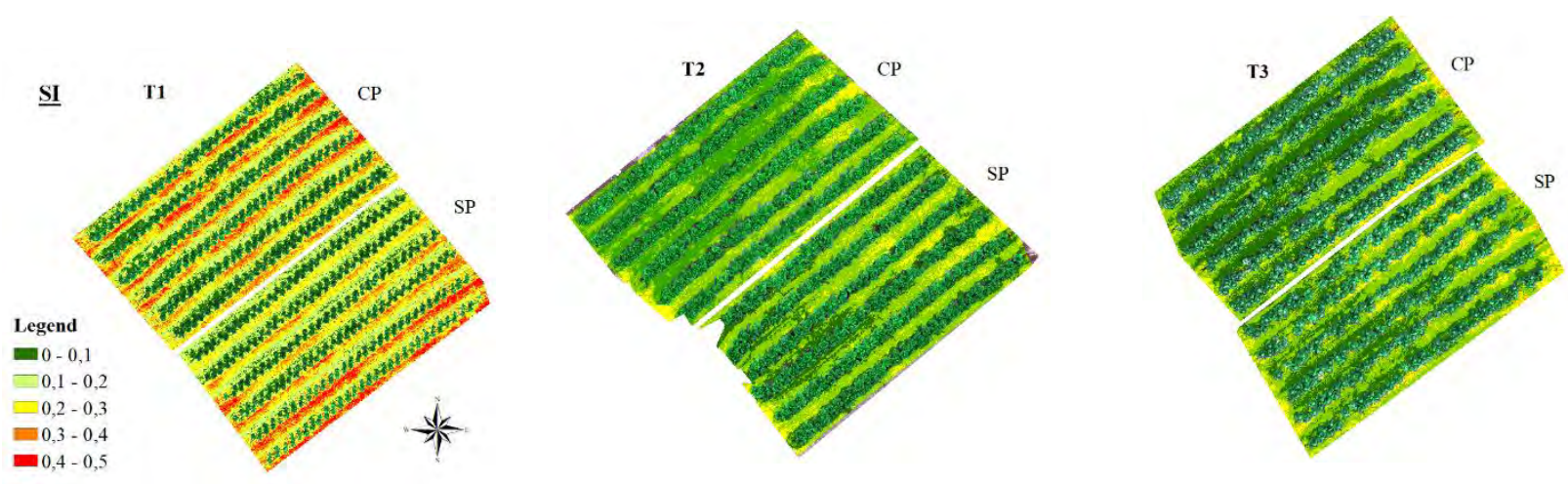

Figura 4. Mapas del SI aplicados solo al suelo desnudo para T1 (11/12/2019), T2

(12/02/2019) y T3 (16/12/2019). 


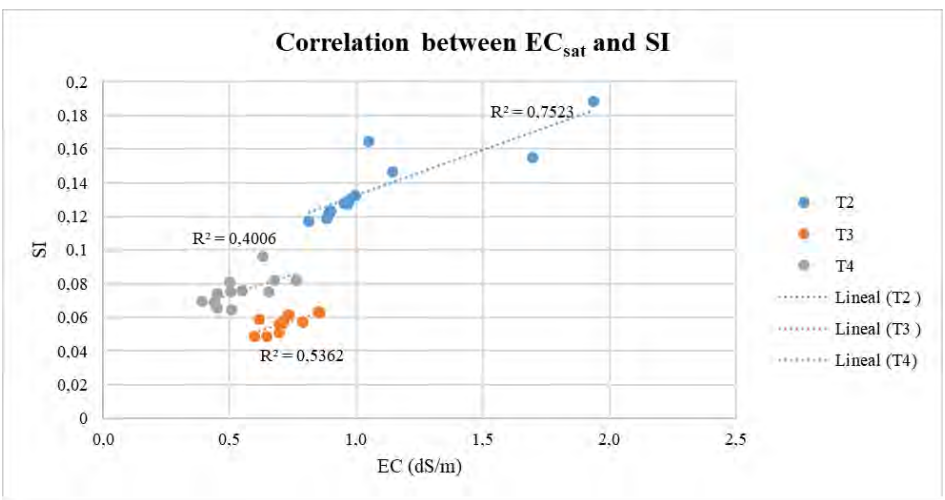

Figura 5. Correlación lineal entre el ECsat y el SI para T2 (20/11/2019), T3 (02/12/2019)

$$
\text { y T4 (16/12/2019). }
$$
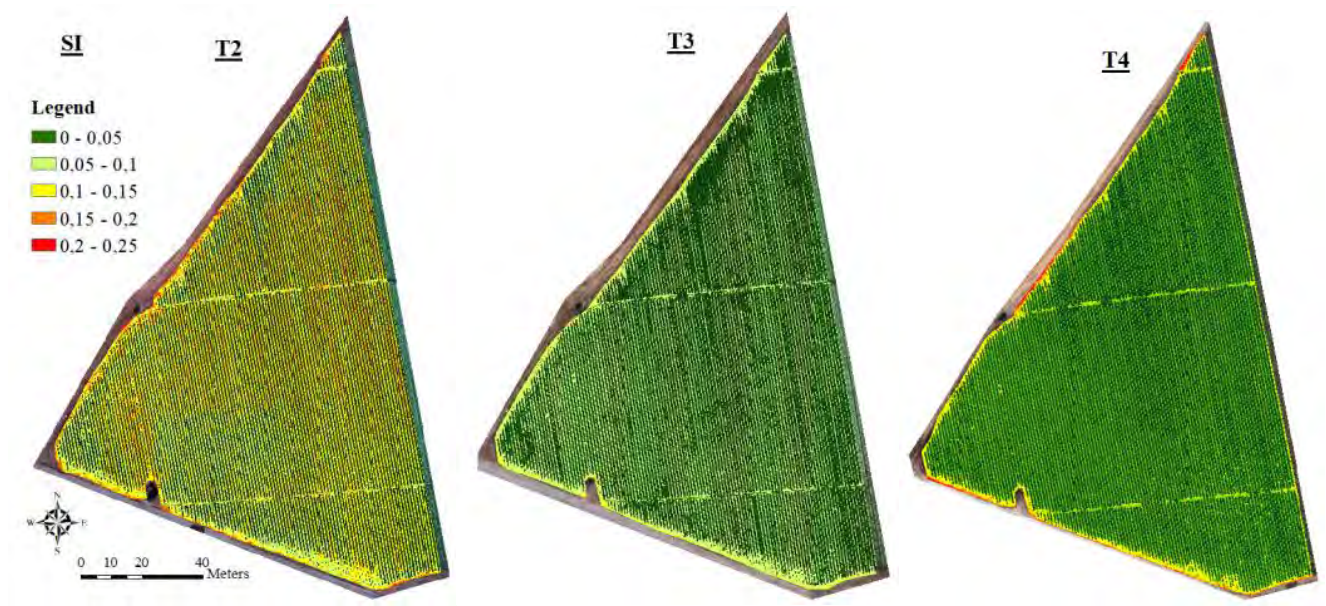

Figura 6. Mapa del SI aplicado al suelo desnudo para T2 (20/11/2019), T3 (02/12/2019) y T4 $(16 / 12 / 2019)$

El agua de riego salina afectó claramente al desarrollo del cultivo y el contenido mineral foliar, viéndose afectadas por diferentes elementos fitotóxicos (datos no mostrados). Tras la recolección, se observó que el peso fresco del cogollo fue un $18 \%$ mayor en el tratamiento CP con respecto en el SP, mientras que no hubo diferencias significativas en el diámetro y la altura del cogollo (datos no mostrados). Otros estudios encontraron diferencias similares entre las lechugas regadas con un ECw de 0.9 y 3.6 dS / m (Di Mola et al., 2018).

\section{Conclusiones y Recomendaciones}

Las altas correlaciones obtenidas con la $\mathrm{T}^{\mathrm{a}}$ de la canopia en comparación con estudios anteriores corroboran la teoría de que las correlaciones con la salinidad del suelo serán mayores con un cultivo más sensible a las sales en el suelo. Como en estudios anteriores también se obtuvieron buenos resultados, se puede concluir que el SI es un índice fiable para predecir la salinidad del suelo. Además, en este experimento se comprobó que el agua de riego no convencional utilizada en el tratamiento $\mathrm{CP}$, del prototipo de tratamiento, demostró ser perfectamente adecuada para el cultivo de lechuga. Por lo tanto, se recomienda la realización de estudios similares con los índices estudiados en condiciones de clima árido o 
semiárido, con suelos y cultivos sensibles a la salinidad y en superficies planas (sin caballón) para ver el movimiento de las sales. Por último, asegurarse de que los vuelos con drones se realicen en condiciones climáticas óptimas, especialmente cuando la humedad relativa no es muy alta, de moda que permitan en un futuro próximo, desarrollar una ecuación / modelo para predecir la salinidad del suelo con una alta precisión a partir de los índices estudiados.

\section{Agradecimientos}

Este trabajo no podría haberse realizado sin el apoyo económico del proyecto de investigación 'Use of Advanced information technologies for Site-Specific management of Irrigation and SaliniTy with degraded water' (ASSIST) financiado por la Fundación SENECA en el Programa Regional "SAAVEDRA FAJARDO", y el Proyecto SHui, cofinanciado por el Proyecto de la Unión Europea GA 773903 y el MOST de China.

\section{Referencias}

Abbas, A., Khan, S., Hussian, N., Hanjra, M.A. \& Akbar, S. (2013). Characterizing soil salinity in irrigated agriculture using remote sensing approach. Physics and Chemistry of the Earth, 55-77, 43-52.

Allbed, A., Kumar, L. \& Aldakheel, Y.Y. (2014). Assessing soil salinity using soil salinity and vegetation indices derived from IKONOS high-spatial resolution imageries: Applications in a date palm dominated region. Geoderma, 230-231, 1-8.

Di Mola, I., Rouphael, Y., Ottaiano, L., Duri, L.G., Mori, M. \& De Pascale, S. (2018). Assessing the effects of salinity on yield, leaf gas exchange and nutritional quality of spring greenhouse lettuce. International Symposium on New Technologies for Environment Control, EnergySaving and Crop Production in Greenhouse and Plant Factory, 479-484.

Gorji, T., Serte, E. \& Tanik, A. (2017). Monitoring soil salinity via remote sensing technology under datascarce conditions: A case study from Turkey. Ecological Indicators, 74, 384-391.

Ivushkin, K., Bartholomeus, H., Bregt, A.J. \& Pulatov, A. (2017). Satellite thermography for soil salinity assessment of cropped areas in Uzbekistan. Land Degradation \& Development, 28, 870-877.

Ivushkin, K., Bartholomeus, H., Bregt, A.J., Pulatov, A., Bui, E.N. \& Wilford, J. (2018). Soil salinity assessment through satellite thermography for different irrigated and rainfed areas. International Journal of Applied Earth Observation and Geoinformation, 68, 230-237.

Lhissou, R., El Harti, A. \& Chokmani, K. (2014). Mapping soil salinity in irrigated land using optical remote sensing data. Eurasian Journal of Soil Science, 3, 82-88.

Squires, V.R. \& Glenn, E.P. (2004). Salinization, desertification and soil erosion. In V.R. Squires (Ed.), The Role of Food, Agriculture, Forestry and Fisheries in Human Nutrition. Oxford: EOLSS Publishers.

Tian, F., Hou, M., Zhang, T. \& Yuan, Y. (2020). Salinity stress effects on transpiration and plant growth under different salinity soil levels based on thermal infrared remote (TIR) technique. Geoderma, 357, 113961. 